\title{
EQUILIBRIUM OF INVERSE-DISTANCE FORCES IN THREE-DIMENSIONS
}

\author{
T. S. Motzkin and J. L. WALSH
}

\begin{abstract}
This paper is about the field of force in three dimensions due to particles that repel according to the inverse-distance law, the analog of the field in the complex plane used by C. F. Gauss in studying the zeros of the derivative of a polynomial. In particular it deals with the analog in three dimensions of Jensen's theorem and related theorems in the plane, shows that the positions of equilibrium in the field of force when the total mass is zero are invariant under spherical transformation, and gives a geometric construction to find the force due to an axially symmetric distribution of matter.
\end{abstract}

To be more explicit, it is classical that C. F. Gauss indicated that in the complex plane the zeros of the derivative $p^{\prime}(z)$ of a polynomial $p(z)$ (not multiple zeros of $p(z)$ ) are precisely the positions of equilibrium in the field of force due to particles at the zeros of $p(z)$, where each particle $z_{0}$ has as mass the multiplicity of $z_{0}$ as a zero of $p(z)$, and where each particle repels with a force equal to its mass times the inverse distance. Gauss's theorem has been widely used in the study of the location of the zeros of $p^{\prime}(z)$ by $F$. Lucas, Bôcher, Jensen, and later writers [see for instance Marden, 2; Walsh, 6]. Also in three-dimensions, G. Sz. Nagy [4] has introduced the concept of distance polynomial, namely as

$$
F(x, y, z) \equiv C \prod_{1}^{n}\left[\left(x-x_{k}\right)^{2}+\left(y-y_{k}\right)^{2}+\left(z-z_{k}\right)^{2}\right], \quad C>0,
$$

whose derivative is defined

$$
F^{\prime}(x, y, z) \equiv \sum_{1}^{n}\left(F_{x}^{2}+F_{y}^{2}+F_{z}^{2}\right) / 4 F
$$

when the $n$ points $P_{k}:\left(x_{k}, y_{k}, z_{k}\right)$ are given. Sz. Nagy then sets up the Gaussian field of force, namely a set of (possibly multiple) particles at the respective zeros of $F(x, y, z)$, where each particle repels with a force equal to its mass times the inverse distances; each zero of $F^{\prime}(x, y, z)$ not a multiple zero of $F(x, y, z)$ is then a position of equilibrium in the corresponding field of force.

This field of force may now be regarded as an appropriate object of independent study, and is so regarded in the present note. New results are obtained beyond those already in the literature. Applications to distance polynomials are immediate, and are left to the reader. 
F. Lucas's theorem asserts that the smallest convex set of the plane containing the zeros of a nontrivial polynomial $p(z)$ contains also the zeros of $p^{\prime}(z)$. The analog clearly holds in higher dimension, as Sz. Nagy indicates, and the results that follow are complementary to the analog of Lucas's theorem.

1. An extension of Jensen's theorem. We recall the statement of Jensen's theorem:

THEOREM 1 (Jensen's theorem). Let $p(z)$ be a nonconstant real polynomial, let all circles (Jensen circles) be constructed, each having as diameter the line segment joining a conjugate pair of zeros of $p(z)$. Then each nonreal zero of the derivative $p^{\prime}(z)$ lies in the closed interior of a Jensen circle.

The first published proof of Jensen's theorem was given by Walsh [6], who also laid the foundation for the deeper study of the zeros of the derivative of a real polynomial. Under the hypothesis of Jensen's theorem, the force in the Gaussian field due to two equal conjugate imaginary particles has [Walsh, $7, \S 1.4 .1]$ a nonzero component toward the axis of reals, parallel to that axis, or away from that axis at every non-real point respectively interior to the Jensen circle, on the Jensen circle, or exterior to the Jensen circle. Thus at each non-real point $P$ exterior to all Jensen circles the force due to each conjugate pair of particles has a nonzero component away from the axis of reals, and at each such point $P$ the force due to each particle at a real zero has also such a nonzero component, so the total force at $P$ is not zero. Thus $P$ is neither a point of equilibrium in the field of force nor a multiple zero of $p^{\prime}(z)$, hence is not a zero of $p^{\prime}(z)$, and Jensen's theorem is established.

This same proof gives at once:

ThEOREM 2. In three dimensions let there be a finite number of positive Gaussian particles situated symmetrically with respect to the $(x, y)$-plane, and let all (Jensen) spheres be constructed whose diameters are line segments joing pairs of points symmetric in the $(x, y)$-plane. Then each position of equilibrium lies in the closed interior of some Jensen sphere.

Theorem 2 is given by Sz. Nagy [4].

Another result in three dimensions is analogous to a result [Walsh, 7, §2.2.2, Theorem 3] in the plane: 
THEOREM 3. In three dimensions, let a positive distribution of total mass $n$ consist of two particles $(0,0, \pm 1)$ each of mass $k$, and particles of total mass $n-2 k$ in the $(x, y)$-plane. Then each equilibrium point lies either in the $(x, y)$-plane or in the closed sphere whose center is the origin and radius $[(n-2 k) / n]^{1 / 2}$.

In the proof we shall need

Lemma 1. If the force at a point $P$ due to $m$ variable unit particles in a plane $\pi$ not containing $P$ has the direction of a line $\lambda$ through $P$ then the force is not greater than it would be if all $m$ particles were concentrated at the intersection of $\pi$ and $\lambda$.

Denote by $\pi^{\prime}$ the inverse of $\pi$ in the unit sphere $S$ whose center is $P$. The force at $P$ due to a particle at $Q$ is in magnitude, direction, and sense the vector $Q^{\prime} P$, where $Q^{\prime}$ is the inverse of $Q$ in $S$. The force at $P$ due to the $m$ given particles is equivalent to the force at $P$ due to an $m$-fold particle on $\lambda$ situated at the inverse $Q_{0}$ of the center of gravity $Q_{0}^{\prime}$ of the points $Q^{\prime}$, inverses of the given particles $Q$ on $\pi$. Since the points $Q$ lie on $\pi$, their inverses $Q^{\prime}$ lie in $\pi^{\prime}$, and $Q_{0}^{\prime}$ lies on or within $\pi^{\prime}$ and on $\lambda$. The greatest force $Q_{0}^{\prime} P$ is exerted if $Q_{0}^{\prime}$ lies at the intersection (other than $P$ ) of $\pi^{\prime}$ and $\lambda$, which implies that all $m$ particles are concentrated at the intersection of $\pi$ and $\lambda$.

The present Lemma 1 combined with [Walsh, 7, Lemma 2, § 2.2.2] and the method of proof of [Walsh, 7, §2.2.2, Theorem 3] now establishes the present Theorem 3.

2. Second extension of Jensen's theorem. We turn now to an analog of [Walsh, $7, \S 2.5$, Theorem 1]:

THEOREM 4. Let a positive distribution of mass consist of a pair $(0,0, \pm 1)$ of equal particles, plus a distribution of mass in the $(x, y)$ plane in the disk $x^{2}+y^{2} \leqq r$. Then all positions of equilibrium not in the $(x, y)$-plane lie in the spindle-shaped region whose boundary is formed by the rotation about $0 z$ of the shorter arc bounded by $(0,0, \pm 1)$ of the circle through those two points tangent at those points to the lines joining those points to $(r, 0,0)$.

It will be noticed [Walsh, $7, \S 2.2 .1$, Theorem 1] that the circular arc (except for $z=0$ or \pm 1 ) is precisely the locus of points of equilibrium in the $(x, y)$-plane due to the two given particles and to a single particle of suitably variable mass in the point $\left(x_{0}, 0,0\right)$ as $x_{0}>0$ varies. If $P:\left(x_{0}, y_{0}, z\right), z_{0} \neq 0$, is a point exterior to the spindle, then 
the line of force at $P$ due to the two given particles lies in the plane $y_{0} x-x_{0} y=0$ and passes through the point $\left(x_{1}, y_{1}, 0\right)$, where $x_{1}^{2}+y_{1}^{2}>r^{2}$ (loc. cit.). On the other hand, the line of resultant force at $P$ due to the given particles in the $(x, y)$-plane cuts that plane in a point $\left(x_{2}, y_{2}, 0\right)$ where $x_{2}^{2}+y_{2}^{2} \leqq r^{2}$, for that fact is true for the line of force at $P$ due to each given particle in the $(x, y)$-plane. Consequently the total force at $P$ is not zero, and Theorem 4 is proved.

By way of contrast to Theorem 4 and to [Walsh, $7, \S 2.5$, Theorem 1] we now indicate that the replacement of the region $x^{2}+y^{2} \leqq r^{2}$ in the $(x, y)$-plane of Theorem 4 by the region $x^{2}+y^{2} \geqq r^{2}$ in the $(x$, y)-plane gives no diminution in the interior of the Jensen sphere (Theorem 2) as the locus of positions of equilibrium. Let $P$ be a point not in the $(x, y)$-plane interior to the Jensen sphere corresponding to the two equal particles $(0,0, \pm 1)$; we shall show that $P$ is a position of equilibrium in the field of force due to the those particles and a suitable distribution in the $(x, y)$-plane satisfying $x^{2}+y^{2}>r^{2}$. Choose for definiteness $P$ in the $(x, z)$-plane, $z>0$. The force at $P$ due to the two given particles is represented by a certain vector $\lambda$ in that plane with a nonzero negative $z$ component. The inverse in the sphere of radius unity whose center is $P$, of the entire $(x, y)$-plane is a spherical shell $S$ of which $P$ is the point furthest from that plane. The inverse of the region $x^{2}+y^{2} \geqq r^{2}$ is a spherical cap of $S$ containing $P$ and bounded by a circle $\Gamma$ which is the inverse of the circle $x^{2}+y^{2}=r^{2}$ in the $(x, y)$-plane. The spherical cap and the plane of $\Gamma$ bound a closed region $R$ which is a convex subregion of the closed interior of $S$. Any set of particles in the region $x^{2}+y^{2} \geqq r^{2}$ of the $(x, y)$ plane have inverses which lie on the spherical cap, and the corresponding force which they exert at $P$ is represented by a single nonzero vector of corresponding multiplicity whose initial point $Q$ in $R$ is the center of gravity of those inverses and whose terminal point is $P$. Then $Q$ is a point in the convex closed region $R$, and conversely any point $Q$ of $R$ may be chosen as the initial point of the vector $Q P$ by suitably choosing the position and (integral) masses of the distribution in the region $x^{2}+y^{2} \geqq r^{2}$ of the $(x, y)$-plane. In particular the distribution may be so chosen that the corresponding force exerted at $P$ is the negative of $\lambda$, so $P$ is a point of equilibrium, as we have asserted.

Theorems 3 and 4 are in a sense mutually complementary; each limits the possible point sets on which the equilibrium points are situated, the one based on magnitude of the masses on and off the $(x, y)$-plane, the other based wholly on the position of those masses; they both apply in any specific case when the masses at $(0,0, \pm 1)$ (supposed to be the only masses exterior to the $(x, y)$-plane) and those in the $(x, y)$-plane are given. 
Of course the analog of Lucas's theorem is available too, for the purpose of further limiting the possible point sets where the equilibrium points are located; thus if a closed dihedral angle less than $\pi$ contains all masses, it contains also all equilibrium points.

With the hypothesis of Theorem 3 , a point $P:\left(x_{1}, y_{1}, z_{1}\right)$ not $(0,0, \pm$ 1) but on the surface of the sphere mentioned, is a point of equilibrium when and only when all the $(n-2 k)$ units of mass in the $(x, y)$-plane are concentrated at the point of the $(x, y)$-plane where the tangent line at $P$ to the circle through $P$ and $(0,0, \pm 1)$ cuts the $(x, y)$-plane; compare [Walsh, 7, §2.2.1].

Likewise, with the hypothesis of Theorem 4 , a point $P$ of the surface of the spindle (other than $(0,0, \pm 1)$ is an equilibrium point when and only when the mass in the $(x, y)$-plane is concentrated in a single point in the $(x, y)$-plane on the circle $x^{2}+y^{2}=r^{2}$, in the plane through $O z$ and $P$, and that mass has a single suitable value (not necessarily integral) depending on the masses at $(0,0, \pm 1)$.

3. Transformation of force field. The study of the location of critical points of a polynomial may be interpreted as a chapter of circle geometry, and the study of the location of equilibrium points in three dimensions as a chapter of sphere geometry. Circle and sphere transformations may be a convenient tool in the respective theories, as we now indicate. The following theorem is essentially due to Bôcher (1904) for two dimensions; we use the the phraseology for three dimensions.

THEOREM 5. In any finite number of dimensions, the direction of the force (including sense) in a field due to a finite number of positive and negative particles of total mass zero which repel according to the inverse distance law is invariant under spherical tranformation.

If such a field of force is given, and the total mass is not zero, we may adjoin suitable particles at infinity so that the total mass becomes zero. These new particles then persist under a spherical transformation that carries the point at infinity into a finite point.

Let $P$ be a point in the field of force due to particles $Q_{1}, Q_{2}, \ldots$, $Q_{k}$ of respective positive masses $m_{1}, m_{2}, \cdots, m_{k}$. The force at $P$ due to the particle $Q_{1}$ is represented in magnitude, direction, and sense by the $m_{1}$-fold vector $Q_{1}^{\prime} P$, where $Q_{1}^{\prime}$ is the inverse of $Q_{1}$ in the unit sphere whose center is $P$. The force at $P$ due to all the particles $Q_{i}$ is represented by the sum of the vectors $Q_{i}^{\prime} P$, of respective multiplicities $m_{i}$, where $Q_{i}^{\prime}$ is the inverse of $Q_{i}$ in that same sphere. The total force at $P$ due to the particles $Q_{i}$ is thus represented by a vector $Q_{c}^{\prime} P$ of multiplicity $m_{1}+m_{2}+\cdots+m_{k}=M$ where $Q_{0}^{\prime}$ is the 
center of gravity of the weighted points $Q_{i}^{\prime}$, so the total force at $P$ due to the particles $Q_{i}$ is equivalent to the force at $P$ due to a particle of mass $M$ situated at $Q_{0}$, the inverse of $Q_{0}^{\prime}$ in the same unit sphere.

We introduce the concept of center of gravity with respect to $P$ of the weighted points $Q_{i}$. This shall be the ordinary center of gravity of the $Q_{i}$ when $P$ lies at infinity, and otherwise is defined to be invariant under any spherical transformation, in particular under a transformation that carries $P$ to infinity and carries the $Q_{i}$ into finite points. It will be noted that the point $Q_{0}$ previouly defined is precisely the center of gravity with respect to $P$ of the given points $Q_{i}$.

If now the field of force of Theorem 5 is due to $k$ positive particles at points $Q_{i}$ of respective masses $m_{1}, m_{2}, \cdots, m_{k}$, and to $j$ negative particles $R_{i}$ of respective masses $n_{1}, n_{2}, \cdots, n_{j}\left(n_{i}<0\right)$, where $m_{1}+$ $m_{2}+\cdots+m_{k}=M=-n_{1}-n_{2}-\cdots-n_{j}$, the total force at $P$ is the force due to an $M$-fold particle situated at the center of gravity $Q_{0}$ with respect to $P$ of the $Q_{i}$, plus the force exerted at $P$ by a $(-M)$ fold particle situated at the center of gravity $R_{0}$ with respect to $P$ of the $R_{i}$. The total force at $P$ is thus the force at $P$ due to the positive particle of mass $M$ at $Q_{0}$ plus the force at $P$ due to the negative particle of mass $(-M)$ at $R_{0}$, which is known [Walsh, 7, §4.1.2] to have the direction and sense at $P$ of the circular arc $Q_{0} P R_{0}$, on which $P$ separates $Q_{0}$ and $R_{0}$. Infinity is defined to be a position of equilibrium if it can be carried into such a finite position under spherical transformation of the given masses. The direction of the total force at $P$ (which is zero if and only if $Q_{0}$ and $R_{0}$ coincide) is thus expressed in a form invariant under spherical transformation, and Theorem 5 is established.

An equivalent statement of the theorem is that the lines of force (including sense) are invariant under spherical transformation. Thus the surfaces orthogonal to the lines of force are also invariant. We have too the

CoROLlary. Under the conditions of Theorem 5, positions of equilibrium are invariant under spherical transformation of space.

Positions of equilibrium are points different from the given particles where the force is zero, hence where direction of the force is not defined.

It is obvious that the theorem extends to distributions of matter not in discrete particles, but such that the field of force can be approximated by a field due to such particles. Proofs of Theorem 5 for stereographic projections of the plane are given not merely by Bôcher but also by Marden [2, §11] and Walsh [7, §4.1.2]. 


\section{Axial symmetry. As an application of Theorem 5, we prove}

THEOREM 6. With force proportional to inverse distance in three dimensions, let $C$ be a uniform circumference of center 0 , radius $\rho$, and total mass unity in a plane $\pi$, and let the axis of $C$ be $\alpha$. Then the total force at a point $P$ of $\pi$ interior to $C$ is zero, that at a point $P$ of $\pi$ exterior to $C$ is $1 / \overline{O P}$, and that at a point not in $\pi$ nor $\alpha$ has a nonzero component sensed away from $\alpha$.

At a point $P$ in $\pi$, the logarithmic potential due to mass $C$ is a constant or log $\overline{O P}$ plus a constant according as $P$ is interior or exterior to $C$, for the potential is harmonic and behaves like $\log \overline{O P}$ when $\overline{O P}$ is sufficiently large; thus the force at $P$ is as asserted. If now $P$ lies on one side of $\pi$, let $O_{1}$ be the point of $\alpha$ farthest from $\pi$ on the sphere through $P$ and $C$ and separated by $\pi$ from $P$. Let the line $O_{1} P$ cut $\pi$ at some point $P_{1}$ interior to $C$. The total force, at $P$ is due to $C$ of mass unity and to a particle at infinity of mass minus unity. Invert the space in the sphere $S$ of center $O_{1}$ passing through $C$. Then $O_{1}, C, P, S$ are transformed into $\infty, C, P_{1}$, and $\pi$ respectively. By Theorem 5 , the force at $P_{1}$ due to the transformed mass is proportional to the force (zero) at $P_{1}$ due to $C$, plus the force at $P_{1}$ due to the (negative) particle at $O_{1}$, in direction and sense $P_{1} O_{1}$. Then the original force (reversed in sense by inversion) exerted at $P$ has direction and sense $O_{1} P$, hence has a nonzero component at $P$ directed away from $\alpha$, so Theorem 6 is established.

The proof just given determines the equivalent particle, preserving the force exerted by $C$ at $P$, hence the components of that force too are immediately determined by construction.

In the plane $\pi_{1}$ through $\alpha$ and the same point $P$, the line $P O_{1}$ bisects the angle at $P$ between the two chords $P C_{1}$ and $P C_{2}$ from $P$ through $C$ in $\pi_{1}$, where $C_{1}$ and $C_{2}$ are distinct and lie in $\pi_{1}$ on $C$. Thus the line of force at $P$ has the direction of a hyperbola in $\pi_{1}$ with foci $C_{1}$ and $C_{2}$. We state the

COROLlaRY 1. If a distribution of positive mass $M$ has axial symmetry in a line $\alpha$ and lies in a plane $\pi$ orthogonal to $\alpha$, then there is no position of equilibrium except in $\pi$; at any point not on $\alpha$ nor $\pi$ the force has a nonzero component away from $\alpha$. There is no position of equilibrium in $\pi$ except interior simultaneously to all the circumferences in $M$ having $\alpha$ as axis.

CoRollary 2. If a distribution of positive mass $M$ has axial symmetry in a line $\alpha$ and lies in more than one plane orthogonal to 
$\alpha$, let the planes be in order $\pi_{1}, \pi_{2}, \cdots, \pi_{\nu}$. Then there is no position of equilibrium on $\pi_{1}$ or $\pi_{,}$. Except on $\alpha$ there is no position of equilibrium, for the total force has a nonzero component directed away from $\alpha$.

It is obvious that if a finite number of circular wire distributions of uniform positive masses lie in a plane, and if the corresponding circular dises have a region $D$ in common, then each point of $D$ is a position of equilibrium.

Under the conditions of Theorem 6, each line of force is a hyperbola whose axes are $\alpha$ and a line in $\pi$ through $\alpha$, with force on $C$, which may degenerate to $\alpha$ or a line in $\pi$ through $O$. The orthogonal surfaces to the lines of force are oblate ellipsoids with $\alpha$ as axis of revolution; a degenerate case is the disk of $C$.

There are naturally numerous other geometric results which correspond to known and even to new theorems in the plane, compare [Walsh, 7, Ch. 3]. As simple examples corresponding to new theorems in the plane we mention:

With the inverse distance law, let pairs of equal positive particles mutually inverse in the $(x, y)$-plane lie on or interior to the sphere, $x^{2}+y^{2}+z^{2}=1$, and perhaps other positive particles lie in the $(x, y)$ plane. Then all position of equilibrium lie in the closed interior of the ellipsoid $x^{2}+y^{2}+2 z^{2}=2$.

With the same law, let pairs of equal positive particles mutually inverse in the $(x, y)$-plane lie on or interior to the circunference $n^{2}+$ $z^{2}=1, y=0$, and perhaps other positive particles lie in the $(x, y)$ plane. Then all positions of eqiilibrium lie in the closed interior of the ellipsoid $x^{2}+2 y^{2}+2 z^{2}=2$.

In these respective cases, all closed Jensen spheres for the given particles lie in the closed ellipsoids defined. Indeed, the new theorems just proved determine the actual loci of the position of equilibrium under the several hypotheses on the given particles, now no longer required to be of integral masses.

These ellipsoids are the analogs of the Jensen ellipses, first introduced by J.L.W.V. Jensen. For proofs of the theorems compare [Walsh, 7, 3.8]. The former of these theorems is valid for several given spheres containing particles, and several ellipsoids similar to $x^{2}+y^{2}+2 z^{2}=2$ are constructed; other positive particles are permitted in the $(x, y)$-plane. Lirewise the latter of these theorems is valid for several given circumferences whose centers lie in the $(a, y)$-plane, and ellipsoids are constructed similar to $x^{2}+2 y^{2}+2 z^{2}=2$. Here of course the word similar is used in the technical sense. 
We remark that results in the plane concerning critical points of rational functions [Walsh, 7, Ch. 4 and 5] have as analogs in higher spaces results involving both positive and negative distributions of matter.

5. Extrema of potential. Inverse-distance forces in any number $d$ of dimensions correspond to the logarithmic potential $\log r$. This potential for a positive mass distribution and exterior to it, is superharmonic for $d=1$, harmonic for $d=2$, and subharmonic for $d>2$. Hence its critical points (positions of equilibrium in the corresponding force field) cannot be strong minima for $d \leqq 2$ nor strong maxima for $d \geqq 2$, but may be weak minima or weak maxima for $d=2$. For $d=1$ two positive mass-points produce a maximum on the segment joining them; for two mass-points of unequal sign not of total mass zero, an extremum occurs outside the segment. For a homogeneous circular wire of positive mass in three-space each point of the closed disk bounded by the wire gives a weak global minimum of the potential.

To obtain a strong minimum of the potential for $d=3$ consider the vertices $( \pm 1,0,0),(0, \pm 1,0),(0,0, \pm 1)$ of a regular octahedron as point unit masses. The potential at $(x, y, z)$ is

$$
\begin{aligned}
H(x, y, z) & \equiv \frac{1}{2} \log \left\{\left[(x-1)^{2}+y^{2}+z^{2}\right] \cdot\left[(x+1)^{2}+y^{2}+z^{2}\right] \cdots\right\} \\
& \equiv \frac{1}{2} \log \left[\left(x^{2}+y^{2}+z^{2}+1\right)-4 x^{2}\right]+\cdots,
\end{aligned}
$$

so the first partial derivative of the potential is

$$
\begin{aligned}
H_{x} & \equiv \frac{2 x\left(x^{2}+y^{2}+z^{2}+1\right)-4 x}{\left(x^{2}+y^{2}+z^{2}+1\right)^{2}-4 x^{2}}+\frac{2 x\left(x^{2}+y^{2}+z^{2}+1\right)}{\left(x^{2}+y^{2}+z^{2}+1\right)^{2}-4 y^{2}} \\
& +\frac{\left.2 x^{\prime} x^{2}+y^{2}+z^{2}+1\right)}{\left(x^{2}+y^{2}+z^{2}+1\right)^{2}-4 z^{2}} .
\end{aligned}
$$

Hence $H_{x x}(0,0,0)=(2-4)+2+2=2, H_{x y}(0,0,0)=0, H_{x z}(0,0,0)=$ 0 . Thus $H$ has a strong minimum at $(0,0,0)$.

Since $H(x, 0,0), 0 \leqq x \leqq 1$, has a local minimum at $x=0$ and is - $\infty$ at $x=1$, it must have a maximum at some intermediate point. It is easy to verify (by the formula for $H_{x}$ ) that such a maximum occurs for $x^{2}=1 / 3$ and only there. Obviously this is a point of equilibrium of the force-field, yet not an extremum of the potential, for the latter is subharmonic.

For a mass distribution which is spherically symmetric with respect to a point 0 and lies exterior to some sphere with center 0 , the point 0 must be an extremum of the potential. Hence for $d=2$, the potential, being harmonic, is constant in a neighborhood of 0 . In the 
case of a positive mass distribution, 0 is necessarily a local maximum of the (superharmonic) potential if $d=1$, and a local minimum if $d \geqq 3$.

To study the force-field generated by a homogeneous distribution of total mass one on the surface of the unit sphere, consider a point $(0,0, a), 0<a \neq 1$. The vertical component of the force exerted at this point is

$$
\begin{aligned}
\frac{1}{4 \pi} \int_{S} \frac{a-z}{x^{2}+y^{2}+(a-z)^{2}} d S & =\frac{1}{4 \pi} \int_{-\pi}^{\pi} \int_{-\frac{\pi}{2}}^{\frac{2}{\pi}} \frac{a-\sin \phi}{a^{2}+1-1-2 a \sin \phi} \cos \phi d \phi d \psi \\
& =\frac{1}{2} \int_{-1}^{1} \frac{a-u}{a^{2}+1-2 a u} d u \\
& =\frac{1}{2 a}-\left.\frac{a^{2}-1}{8 a^{2}} \log \left(\frac{a^{2}+1}{2 a}\right)\right|_{-1} ^{1} \\
& =\frac{1}{2 a}-a^{2}-1 \log \frac{|1-a|}{1+a} .
\end{aligned}
$$

For $a<1$, this force is

$$
\frac{a}{1 \cdot 3}+\frac{a^{3}}{3 \cdot 5}+\frac{a^{5}}{5 \cdot 7}+\cdots
$$

Thus when $a$ increases from zero to one, this force increases from zero to one-half. The potential has a single minimum, namely at the center of the sphere. Both force and potential are continuous throughout space; this is in contrast to potential and force due to a circular wire, where the force is not continuous.

\section{REFERENCES}

1. M. Marden, A generalization of a theorem of Bôcher, SIAM Journal of Numerical Analysis, 3 (1966), 269-275.

2. The geometry of the zeros of a polynomial in a complex variable, Math. Surveys, vol. III. Amer. Math. Soc., 1949.

3. G. v. Sz. Nagy, Zur Theorie der algebraischen Gleichungen, Jber. d. deutschen Math.-Verein., 31 (1922), 238-251.

4. ——Über die Lage der Nullstellen eines Abstandspolynoms und seiner Derivierten, Bull. Amer. Math. Soc., 55 (1949), 329-342.

5. A. Schurrer, On the location of the zeros of the derivative of rational functions of distance polynomials, Trans. Amer. Math. Soc., 89 (1958), 100-112.

6. J. L. Walsh, On the location of the roots of the derivative of a polynomial, Annals of Math., 22 (1920), 128-144.

7. - The location of critical points of analytic and harmonic funstions, Amer. Math. Soc. Coll. Pub., 34 (1950).

Received August 10, 1971. Sponsorship: Research supported (in part) by the U. S. Air Force Office of Scientific Research, under Contract AFOSR 1690-69.

University of California, Los ANgELES

AND

UNIVERSITY OF MARYLAND 